\title{
FORGERY DETECTION OF FAKE CURRENCY USING IMAGE DERIVATIVE BASED SEGMENTATION TECHNIQUE
}

\author{
Rosy Mishra \\ Asst. Professor \\ Department of CSE \\ Vikash Institute of Technology \\ Bargarh, Odisha, India \\ Rukshar Bano \\ Department of CSE \\ Vikash Institute of Technology \\ Bargarh, Odisha, India
}

\begin{abstract}
Fake currency is a threat to each country throughout the globe. This paper is centered to detect the fake currency use of the image segmentation technique. Fake foreign money aims in discovering the forgery currency. The first step in image processing is preprocessing of the image involve containing cropping smoothing and adjustment of the image. The second step contains the conversion of the image into a gray coloration image. The conversion is done after image segmentation. The features include removal and mitigation. In the last step, compare the image to the original or the forgery image. India is more vulnerable to this problem which is results in a big hurdle. The empirical simulation is carried out using Rupees 100, 500 note of Indian Currency.
\end{abstract}

Keywords-Feature extraction, image processing, counterfeit currency, HSV.

\section{INTRODUCTION}

Almost every country is struggling with the problem of fake notes. A fake 500 makes the Indian currency system a flood and there is no proper way for a common man to deal with the problem. Therefore the rate of corruption has increased in our country which is a hindrance in the development of the country. The common man became a scapegoat for the practice of fake foreign exchange. Sometimes common persons have to take the blame if he will be caught in such fake currency detection while depositing money in banks.

The purpose of digital image processing is to improve the quality of pictorial information and to facilitate automation. It is the use of computer algorithms to perform image processing on the digital image. It has a lot of

\author{
Rosan Patel \\ Department of CSE \\ Vikash Institute of Technology \\ Bargarh, Odisha, India \\ Madhumita Pradhan \\ Department of CSE \\ Vikash Institute of Technology \\ Bargarh, Odisha, India
}

comprehensive algorithms for input facts and can avoid problems such as noise and signal distortion during processing which is not possible in the case of analog processing. Since images are defined by more than 2D more digital image processing can be modeled in the shape of multidimensional systems. Digital image processing approves the utilization of a lot greater problematic algorithms and as a result, can supply each more trendy common overall performance at effortless responsibilities and the implementation of strategies that can also be impossible with the aid of analog. Image segmentation is a system of dividing a digital image into multiple regions and extracting meaningful regions, recognized as a pixel of interest (ROI) unit, which is treated as an additional pixel. The main objective is to simplify or alternate the depiction of an image that is larger and less complex to analyze. The image segmentation is often used to locate objects and boundaries (lines, curves, etc.) in images. More accurate image segmentation is the method of assigning a label to each pixel in an image such that pixels with the same label share positive characteristics. Image restoration and image enhancement are no longer identical in that the latter is designed to emphasize the features of the image that are additionally desirable to the observer but no longer optimal for creating a sensible document from the scientific factor of view. Image enhancement strategies such as stretching or de-blurring, which are coupled with neighboring objectives, equipped with imaging objectives, make no unexpected use of the technique that produced the image. With image correction, noise can be removed correctly by discarding certain resolutions because it is not consistently suitable in many applications. Image enhancement is the process of enhancing image quality for analysis. 


\section{International Journal of Engineering Applied Sciences and Technology, 2020 \\ Vol. 5, Issue 2, ISSN No. 2455-2143, Pages 396-401 \\ Published Online June 2020 in IJEAST (http://www.ijeast.com)}

The spatial resolution depends on two parameters: the number of pixels of the image and the number of bits required for sufficient intensity, the number of bits called depth. The number of pixels shows that digital image quality is illegal using fake money which is a form of fraud or forgery. Counterfeit money is illegal currency generated without government permission. There are some ill-effects of counterfeiting on society, increase in prices due to more money circulating in the economy.

\section{RESEARCH METHODOLOGY}

\section{A. Load Image}

Initially, the image is loaded into the program using the file selector option Image() draws an image in the display window. Validity is required to ensure that the image is valid and requires placing 3 planes indication that only color images are accepted. If verification is performed properly, the image is saved in a variable. The valid image is then displayed on the axes so that the user can select the correct image. The dimensions of the image are then calculated including rows, columns, and several planes are prepared for further processing to fill in the blanks during later processing, while not affecting the original image. The mask has the same dimensions as the image to be compared.

\section{B. Image Acquisition \& Pre-processing}

The image acquisition stage involves the process of acquiring images by using any imaging sensor. After acquiring or obtaining the image different operations are to be performed to various parts of the image for different vision tasks. This is a mandatory step in any image processing process because without an image no processing is possible. The concept of pre-processing is intended to enhance the visual appearance of images and to manipulate datasets of image processing that typically require earlier than most important information analysis and statistical extraction. Image pre-processing is additionally known as image restoration that defines the correction of degradation noise and distortion that are associated with some stage in imaging processing to maximize the reliability of the optical inspection image. Many filter operations reduce or overcompensate some important images, for which the evaluation will be less complicated or faster.

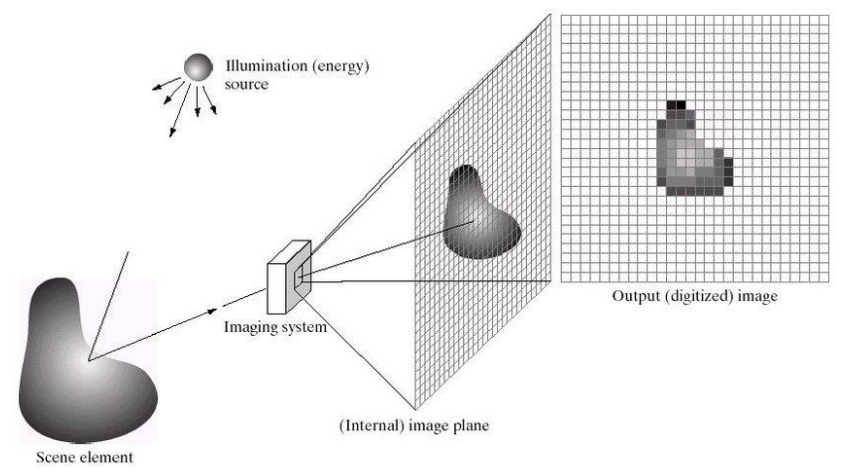

Fig. 1. Image Acquisition and Sampling Model

\section{Gray Scale Conversion -}

Changing a gray image to a gray scale image is changing the RGB value ( $24 \mathrm{bit}$ ) to a gray scale value ( 8 bit). Various image processing techniques and software applications convert a color image into a gray scale image. The acquisition is done in RGB color. It is converted to gray scale because it only creates intensity information that makes processing easier. To process the three components R (red), G (green), B (blue) it takes three RGB values for each pixel and shows the brightness of that pixel as output. One such approach is to average the contribution from each channel:

$$
(\mathrm{R}+\mathrm{B}+\mathrm{G}) / 3
$$

\section{Edge Detection}

Edges that detect changes in intensity can be accomplished using a first or second-order derivative of the local image processing approach format for edge detection. Edge detection algorithms are the title of a set of techniques that intend to identify factors in a digital image processing at which the image is rapidly modified or additionally formalized. The points at which the image brightness adjustment occurs rapidly are usually furnished in a set of edges of curved line segments. Originally edge detection is a derivative-based method that is classified into first-order and second-order derivative filters [5-12]. Firstorder derivative-based filters work better with thick edges while second-order ones give better results with thinner or finer edges.

First-order derivative: It has property 1) which should be zero in the area of constant gray level2) non-zero at the beginning of a gray level step or ramp 3) non-zero along the ramp. A Sober operator is the best example of a firstorder derivative.

Generalized derivative in discrete domain is shown in equation 2.

$$
\frac{d}{d x} f(x)=\lim _{\Delta t \rightarrow 0} \frac{f(x+\Delta x)-f(x)}{\Delta x}
$$

1-D first order derivative is represented in equation 3 as

$$
\frac{\partial f}{\partial x}=f(x+1)-f(x)
$$

2-D first order derivative is represented in equation 4,5 as

$$
\begin{gathered}
\overrightarrow{\nabla f}=\left[\begin{array}{l}
\frac{\partial f}{\partial x} \\
\frac{\partial f}{\partial y}
\end{array}\right] \\
|\overrightarrow{\nabla f}|=\left[\left(\frac{\partial f}{\partial x}\right)^{2}+\left(\frac{\partial f}{\partial y}\right)^{2}\right]^{\frac{1}{2}}=\left|\frac{\partial f}{\partial x}\right|+\left|\frac{\partial f}{\partial y}\right| \\
\frac{\partial f}{\partial x} \cong[f(x+1, y-1)+f(x+1, y+1)+2 f(x+1, y)] \\
-[f(x-1, y-1), f(x-1, y+1)+2 f(x \\
-1, y)]
\end{gathered}
$$




$$
\begin{aligned}
& \frac{\partial f}{\partial y} \cong[f(x-1, y+1)+f(x+1, y+1)+2 f(x, y+1)] \\
&- {[f(x+1, y+1), f(x+1, y-1)+2 f(x, y} \\
&-1)]
\end{aligned}
$$

Second-order derivative: It has property 1) which must have zero in the constant areas. 2) Non-zero at the beginning and end of the gray level step or ramp 3) must be zero with a ramp of constant slops. Laplacian operator is the best example of the first-order derivative.

1-D second order derivative is represented in equation 6 as

$$
\frac{\partial^{2} f}{\partial x^{2}}=f(x+1)+f(x-1)-2 * f(x)
$$

Laplacian is a second order isotropic derivative operator that is mathematically relevant $\mathrm{f}(\mathrm{x}, \mathrm{y})$ to an image function defined as

$$
\begin{gathered}
\nabla^{2} f=\frac{\partial^{2} f}{\partial x^{2}}+\frac{\partial^{2} f}{\partial y^{2}} \\
\frac{\partial^{2} f}{\partial x^{2}}=f(x+1, y)+f(x-1, y)-2 f(x, y) \\
\frac{\partial^{2} f}{\partial y^{2}}=f(x, y+1)+f(x, y-1)-2 f(x, y) \\
\nabla^{2} f(x, y)=f(x+1, y)+f(x-1, y)+f(x, y+1) \\
+f(x, y-1)-4 f(x, y)
\end{gathered}
$$

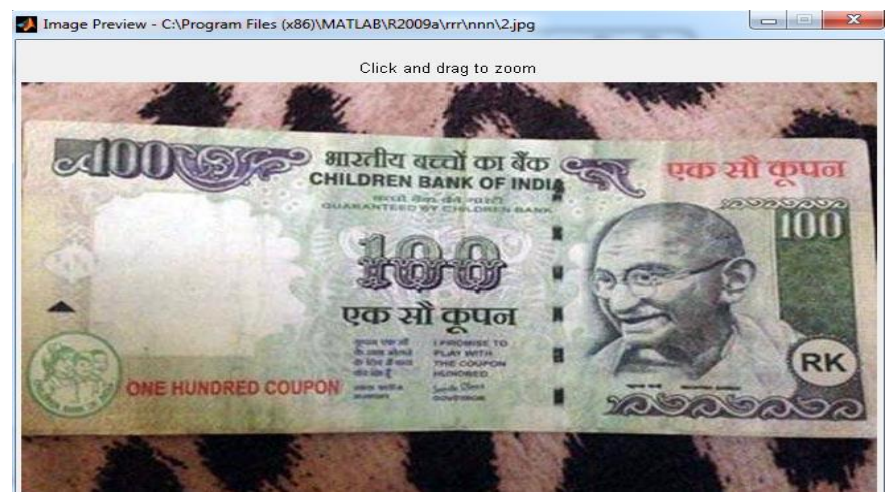

Fig. 2. 100rs Note Input Image

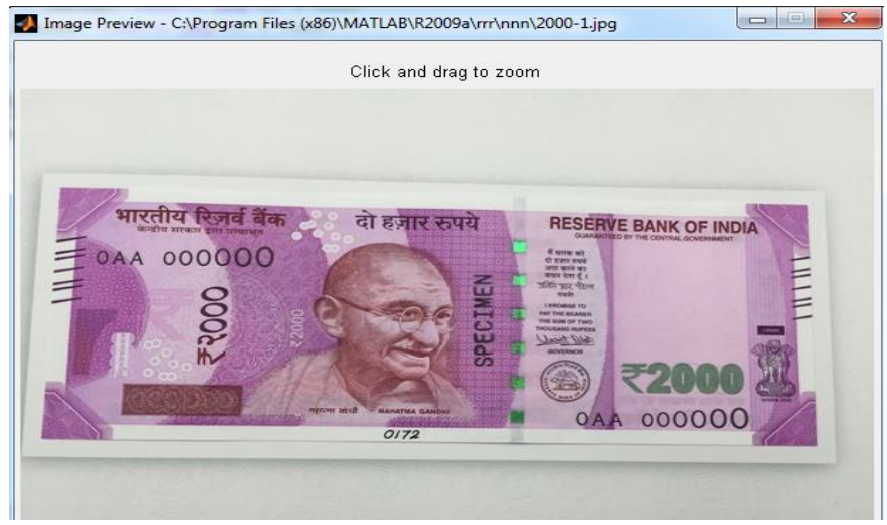

Fig. 3. 2000rs Note Input Image

\section{E. Proposed Architecture}

The proposed system architecture is divided into two parts viz.
1) Currency Recognition
2) Currency Verification

The recognition of the currency usually used the link ID mark and optical variable link of the features. The input is an image or scanned image given to a system that can be of .png and the output tells whether the currency is real or not. The pixel value is calculated for every process. Sometimes watermark is also verified by currency verification. Indian currency which included a watermark security thread. Fluorescence and latent image are used for currency verification. Figure 4 below describes the flowchart for currency recognition. For verification, each segment of the character is extracted and then the pixel value is calculated.

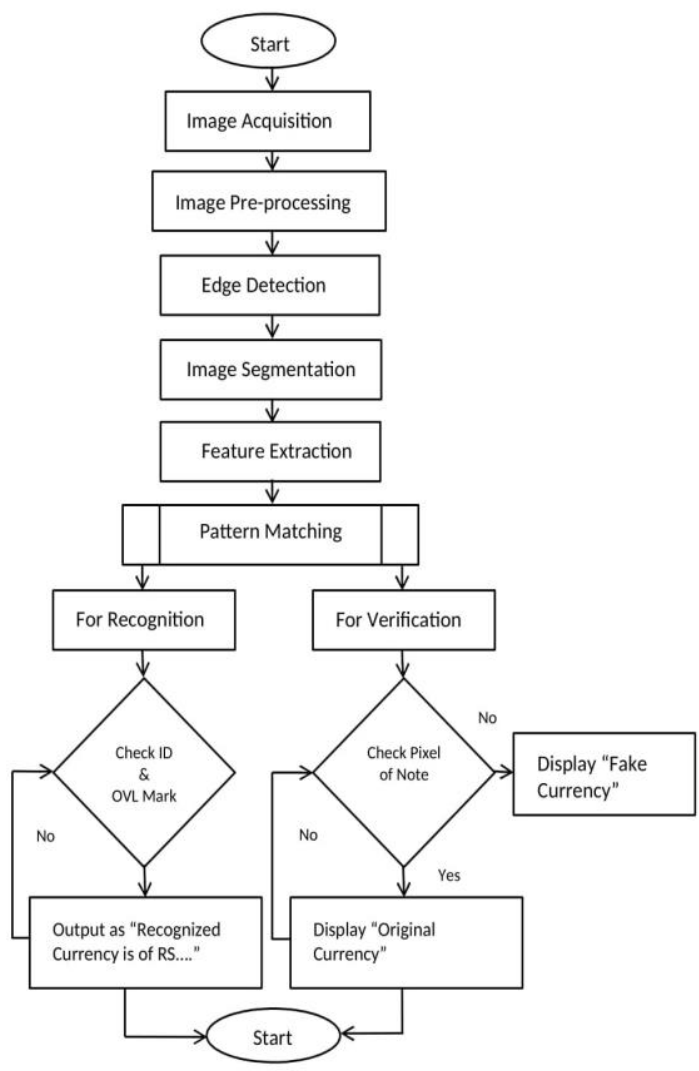

Fig. 4. Proposed Model

Typically, the image is taken in Rgb color, then it is changed to gray scale because it is directly aware of a technique in preference to processing the three components $\mathrm{R}$ (red), G(green), B(blue) makes it easy. RGB value for each pixel. And the output is the brightness of that pixel. A good approach is to take a common contribution from each channel: $(\mathrm{R}+\mathrm{B}+\mathrm{G}) / 3$ however, since perceived brightness often dominates with a different "human-oriented" with the help of inexperienced component and technique weighted average it occure. Have to Take,

e.g.: $0.3 R+0.59 G+0.11 B$. 


\section{International Journal of Engineering Applied Sciences and Technology, 2020 \\ Vol. 5, Issue 2, ISSN No. 2455-2143, Pages 396-401 \\ Published Online June 2020 in IJEAST (http://www.ijeast.com)}

Conversion from RGB to HSV: Hue $(\mathrm{H})$ specifies a pure color (purp, yellow, red, etc.) that provides saturation (S) to provide a degree of saturation. Pure color is diluted with white light. The color value (V), also called its brightness, varies from 0 to 1 . This indicates how dark the color is. Avalue of Ois black, but moving from black to white will increase lightness. HIS component values from RGB component values can be generated using equations 9 and 10 .

$$
\begin{gathered}
\quad H= \begin{cases}\theta & \text { if } B \leq G \\
360-\theta & \text { if } B>G^{\prime}\end{cases} \\
\text { Where } \theta=\cos ^{-1}\left\{\frac{\frac{1}{2}[(R-G)+(R-B)}{\left[(R-G)^{2}+(R-B)(G-B)\right]^{\frac{1}{2}}}\right\} \\
S=1-\frac{3}{(R+G+B)}[\min (R, G, B)], \\
I=\frac{1}{3}(R+G+B) \\
V_{\text {sat }}=1.0-\frac{0.8 V}{255}
\end{gathered}
$$

HSV converted image undergone for image segmentation by removing small object component from the edged binary image.

Gary values of the pixels are represented by subthreshold situation and the rest with hues. Feature generation is used in making an approximation of the color of each pixel by using the thresholding phenomena. On the other hand feature generated by RGB color space is approximated by considering the hue which poses higher order bits [10]. The best method for image analysis is image segmentation which can also be applied here. On the other hand, histogram helps in maintaining the uniform color transition which helps in window based smoothing retrieval. To find the best colour histogram of an image all the higher order bits i.e. $\mathrm{N}$ of red, green, blue of RGB color space are to be linked together. At the final stage this result can be compared with those which are generated using RGB color space.

\section{SIMULATION AND RESULTS}

Empirical simulation using MATLAB 13 with a $2.6 \mathrm{GHz}$ i3 processor.

\section{A. Detect Indian Currency Note}

$\mathrm{BW}=\mathrm{im} 2 \mathrm{bw}$ (I level) converts gray scale image 1 to binary image BW. And in this input image, all pixels are replaced with a brightness higher than the level of value 1 (White) and then all other pixels are replaced with value 0 (black). This variation is related to the possible signal levels for the class of the image. Therefore it can be said that a level value of 0.5 corresponds to an intensity value halfway between the minimum and maximum value of the square.

areaopenReal = bwareaopen $($ BWImageCloseReal, 15);

subplot $(1,2,1)$;

imshow(areaopenReal);

title('Real');

subplot(1,2,2);
areaopenFake $=$ bwareaopen $($ BWImageCloseFake, 15); imshow(areaopenFake);

title('Fake');

\section{B. Count the Number of Black Line}

Counting the black lines is the final step that honestly counts the number of black marks in each image. If a black line is seen completely, indicating that the bank note is real, but if it crosses the value i.e. greater than 1 then that skill is fake. We can use the BW label and use another parameter to remember how many objects we have. In other phrases as we understand the actual note has just one line while the fake note has more than one. You have to play with this code which depends on what bank note you need to do this job but it is someplace to start.

[L,countReal $]=$ bwlabel $($ BWImageCloseReal $)$;

[L,countFake $]=$ bwlabel(BWImageCloseFake);

[L,countFake2] = bwlabel(BWImageCloseFake2);

$\operatorname{disp}([$ 'The total number of black lines for the real note is: 'num2str(countReal)]);

$\operatorname{disp}([$ 'The total number of black lines for the fake note is: num2str(countFake)]);

$\operatorname{disp}([$ 'The total number of black lines for the second fake note:' num2str(countFake2)]);

The image is taken from the saved database for processing of the image morphologically. The process of segmenting a digital image into a large number of regions is formed " 100 rupee note image acquisition". By performing segmentation background objects are extracted. Edge-based segmentation is useful in this process. The process of segmentation is given in figures 5 and 6 below. The image after partition comes with noise and the image taken is an Indian pose. For example, if the triangle is outside, then the money value is 100rs. The OVL link is similarly located and it is decimal value is 100 rs note.
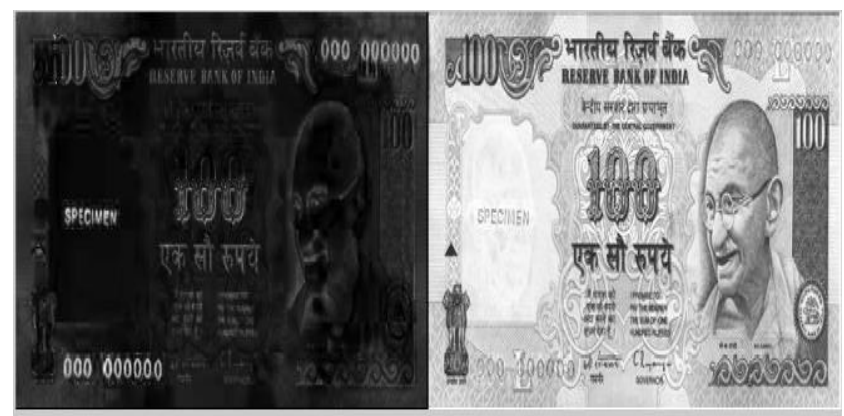

Fig. 5. 100rs Hue-Saturation-Value Image (Real)

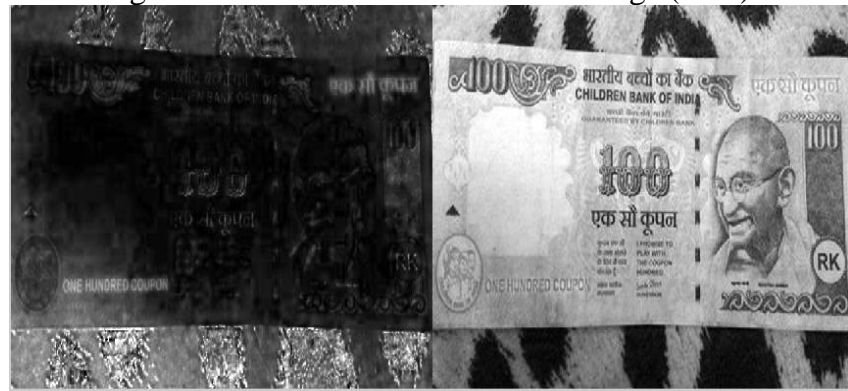

Fig. 6. 100rs Hue-Saturation-Value Image (Fake) 


\section{International Journal of Engineering Applied Sciences and Technology, 2020 \\ Vol. 5, Issue 2, ISSN No. 2455-2143, Pages 396-401 \\ Published Online June 2020 in IJEAST (http://www.ijeast.com)}

The Indian currency was taken after extraction results in character from intaglio printing. This is Figure 7 this is called character extraction of intaglio printing. Currency identification includes features such as ID markings. And it can be identified based on ID mark detection and currency size. For example, if the triangle is outside, then the money value is 2000rs. The OVL link is similarly located and its decimal value is 2000 rs note.

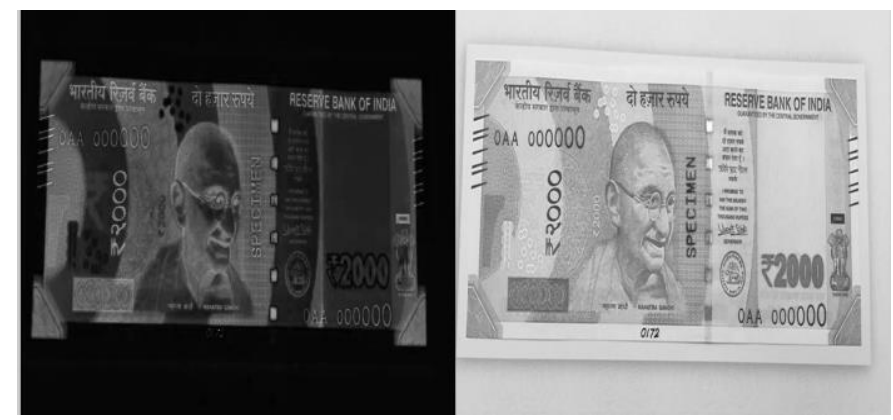

Fig. 7. 2000rs Hue-Saturation-Value Image (Real)

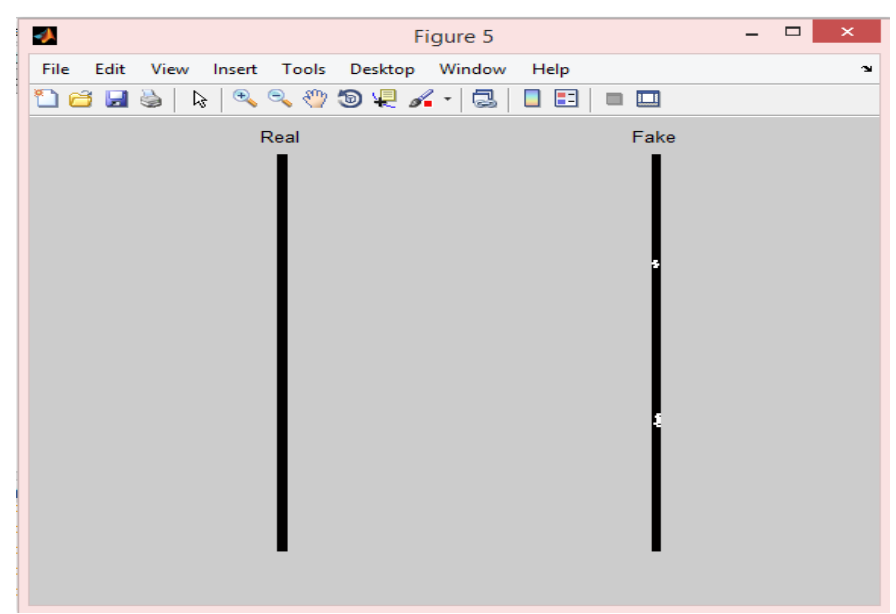

Fig. 8. 100rs Pixels image

The total number of black lines for the real note is: 0 The total number of black lines for the fake note is: 2 $f x>$

Fig. 9. Output image 1

Nothing the thin black stripe of the impure note, you will see that each black line is separated by an exceptionally few pixels at the same time because the real note has no separation i.e. the two are no longer the same. However, you can also see that there are still some components of the line that can be disconnected for the duration of the actual bar above. Like, let's try to connect the line. This is safe because if we do this on the fake image then some parts of the stripe should be separated as the final should not be differentiated but it will help in our actual image analysis. Like, I closed these images through a 6-pixel line that is vertical. Here is the code to do this:

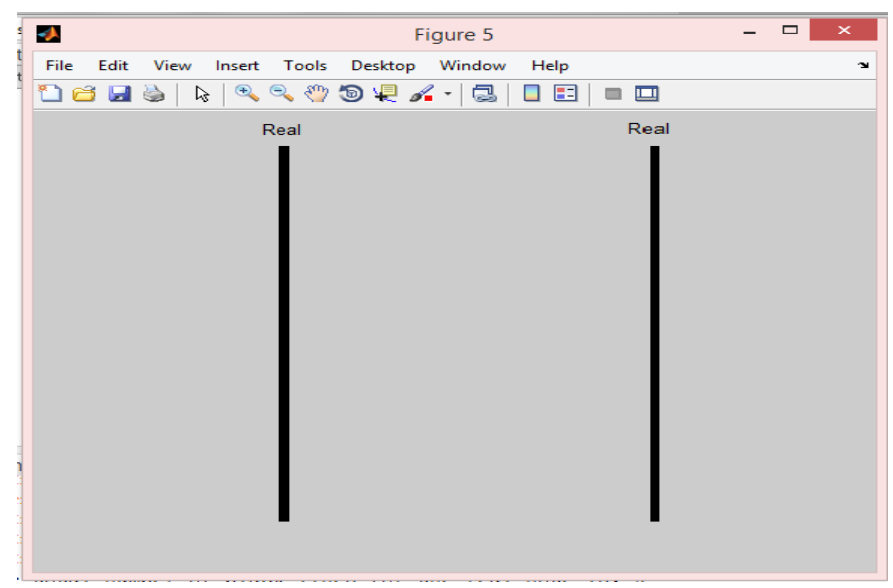

Fig. 10. 2000Rs. Pixels Image

The total number of black lines for the real note is: 0

The total number of black lines for the real note is: 0 $f x>$

Fig. 11. Output Image 2

\section{CONCLUSION AND FUTURE WORK}

Further analysis of currency can be performed if a more accurate and efficient method will be found. MATLAB is used to solve the problem. Day by day research work is developing in this discipline and several image processing methods are carried out so $s$ to get the greater correct result. The proposed computing device is labored efficaciously for extracting attribute of Indian currency pix that capacity solely Indian fake currency is located and recognized. Extracted competencies of currency image will be the use of for Indian money cost recognition as properly as for its verification. The application-based device will be designed to get proper results whether or now not the currency image is faux or it is genuine. Here we have taken only Indian currency as proof but other analyses will recognize other currency also.

\section{REFERENCES}

[1] Alekhya D., Prabha G.DeviSurya, Durga Rao G.Venkata "Fake Currency Detection Using Image Processing and Other Standard Methods". International Journal of Research in Computer and Communication Technology, Vol 3, Issue 1, 2014.

[2] Mudassir Mohd, Ismail Mohd and Mr Ashutosh. B. Makone "An Automated Recognition of Fake Destroyed Indian Currency Notes Using Image Processing". International Journal of Engineering Science \& Research Technology 2014.

[3] M.Deborah and Prathap Soniya "Detection of Fake currency using Image Processing”. IJISETInternational 
Journal of Innovative Science, Engineering \& Technology, Vol. 1, Issue 10, 2014.

[4] Mirza Rubeena and Nanda Vinti "Design and Implementation of Indian Paper Currency. Authentication System Based on Feature Extraction by Edge Based Segmentation Using Sobel Operator". International Journal of Engineering Research and Development, Volume 3, Issue 2, 2012.

[5] Barik Ram Chandra, Mishra Rosy (2016) "Comparative Analogy on Classification and Clustering of Genomic Signal by a Novel Factor Analysis and F-Score Method". In: Artificial Intelligence and Evolutionary Computations in Engineering Systems. Advances in Intelligent Systems and Computing, Vol 394. Springer, New Delhi.

[6] Barik Ram Chandra, Pati R. and Behera H.S.," Robust signal processing compression for clustering of speech waveform and image spectrum", IEEE International Conference on Communication and Signal Processing, April 2-4, 2015, India.

[7] Agrawal Nisha, Urma Sanjukta, Padhan Sonam , Barik Ram Chandra, "Indian Agro Based Pest Region Detection by clustering and Pseudo-Color Image Processing" in International Journal of Engineering Research \& Technology (IJET) ISSN:2278-0181.

[8] Agrawal S., Raidu B., Agrawal K., Barik Ram Chandra, "Prediction of Cancerous Cell by Cluster Based Biomedical CT Image and Analysis", IOSR Journal of Dental and Medical Sciences (IOSR-JDMS), Volume 16, Issue 2 Ver. IV (February. 2017), PP. 67-73.

[9] Barik Prabhat, Barik Ram Chandra, "Feed Forwarded CT Image Registration for Tumour and Cyst Detection using rigid transformation with HSV color segmentation" International Journal of computational System and Engineering, 2019 Vol.5 No.5/6, pp.277 286.

[10] Sahoo Rajat Kumar, Panda Ritu, Barik Ram Chandra, Panda Samrendra Nath, "Automatic Dead Zone Detection in 2-D Leaf Image Using Clustering and Segmentation technique", International journal of image, Graphics and Signal Processing (IJIGSP), vol.10, No. 10, PP. 11-30,2018.DOI: 10.5815/ijigsp. 2018.10.02

[11]Chaple Girish N., Daruwala R.D. , Gofane Manoj S. "Comparisons of Robert, Prewitt, Sobel operator based edge detection methods for real time uses on FPGA", IEEE Conference (ICTSD-2015), Feb. 04 - 06, 2015, Mumbai, India.

[12].Yadollahi M. and Prochazka A., "Image Segmentation for Object Detection", Available at: http://dsp.vscht.cz/konference_matlab/MATLAB11/pri spev ky/129_yadollahi.pdf 